\title{
A Pop Eye Mucosal Bulge in the Duodenal Bulb
}

\author{
Tian-Yin Chen ${ }^{1}$, Xiao-Cen Zhang ${ }^{1}$, Ming-Yan Cai ${ }^{1}$, Xiao-Wen Ge ${ }^{2}$, Ping-Hong Zhou ${ }^{1 *}$ \\ ${ }^{1}$ Endoscopy Center and Endoscopy Research Institute, Zhongshan Hospital, Fudan University, Shanghai, China \\ ${ }^{2}$ Pathology Department, Zhongshan Hospital, Fudan University, Shanghai, China
}

\begin{abstract}
Received: March 09, 2016; Accepted: March 23, 2016; Published: May 03, 2016
*Corresponding author: Ping-Hong Zhou, Endoscopy Center and Endoscopy Research Institute, Zhongshan Hospital, Fudan University, 180 FengLin Road, Shanghai, 200032, P. R. China. Phone: (+86)-21-64041990. E-mail: zhou.pinghong@zs-hospital.sh.cn
\end{abstract}

\begin{abstract}
Neoplasms and malignancies are seldom seen in the duodenum in comparison with other parts of the alimentary tract. Duodenal subepithelial lesions with umbilication are rare, often regarded typical for ectopic pancreas. Meanwhile, premalignant/ malignant transformation of ectopic gland might even be rarer. We present a case of a duodenal intraepithelial neoplasia, most likely originated from an ectopic pancreas, hidden under normal epithelium of duodenum; with a "pop eye" left on it's the surface.
\end{abstract}

Keywords: Duodenal ectopic pancreas; Intraepithelial neoplasia

\section{Introduction}

Neoplasms and malignancies in the duodenum are relatively rare in comparison with other parts of the alimentary tract. Duodenal subepithelial lesions usually include ectopic pancreas, lipomas, gastrointestinal stromal tumors, lymphoma and etc. Non-ampullary lesions present with inconspicuous symptoms and are often discovered during a routine endoscopy, while ampullary lesions may have an early onset of jaundice and pancreatitis due to the obstruction of the duodenal papillary orifice. Endoscopic techniques such as high-definition white-light endoscopy (HWE), narrow band imaging (NBI), and confocal laser endomicroscopy (CLE) have been applied to detect alimentary lesions and distinguish from cancerous tumors [1]. We report a case of a duodenal tubulovillous adenoma, probably originated from an ectopic pancreas, presenting with an appearance of pop eye mucosal bulge.

\section{Case Report}

Upper GI endoscopy in a 58-year-old man who complained of chronic upper abdominal discomfort revealed a $2.0 \mathrm{~cm}$ bulge in the duodenal bulb (Figure 1). There were no specific findings in his past and family history. Laboratory analysis came back negative. Abdominal CT scan ruled out lesions or metastasis in other places. The bulged lesion had smooth surface mucosa and an orifice oozing clear fluid at the top. It was later removed with endoscopic piecemeal mucosal resection (EPMR), and the specimen had a jelly-like texture (Figure 2). HE section showed tubulovillous adenoma with middle-high grade dysplasia, oxyphilic type (Figure 3). Immunohistology showed KI67
$(30 \%+)$, MUC5 (+), MU6 (+), CK7 (+), CK20 (-) (Figure 3), leading to a diagnosis of intraepithelial neoplasia originating from ectopic gland (most likely biliary-pancreatic duct). The patient had no fever, abdominal infection, pancreatitis or other complications after EPMR and was discharged after 3 days.

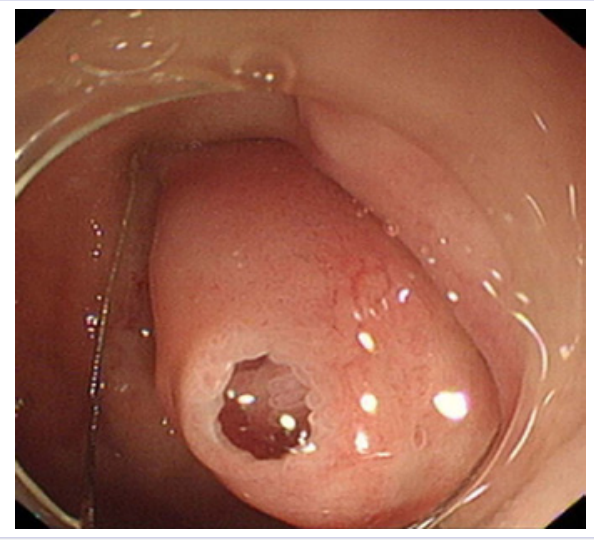

Figure 1: The lesion presented with a $2.0 \mathrm{~cm}$ bulge in the duodenal bulb. The bulged lesion had smooth surface mucosa and an orifice oozing clear fluid at the top.

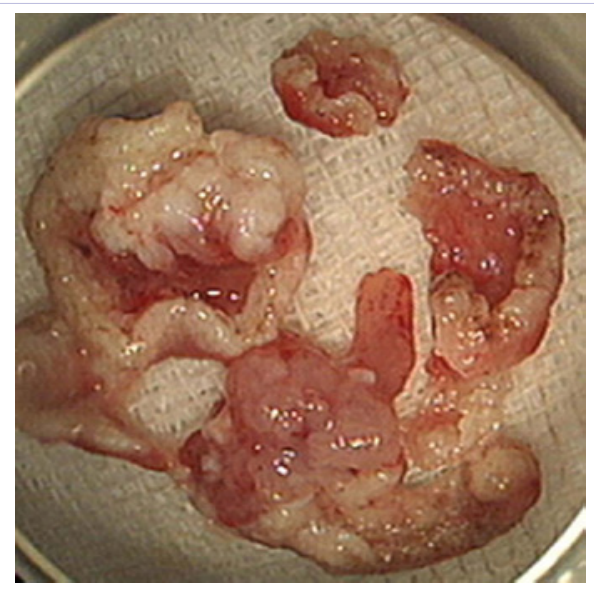

Figure 2: The lesion was resected by endoscopic piecemeal resection and the specimen had a jelly-like texture. 


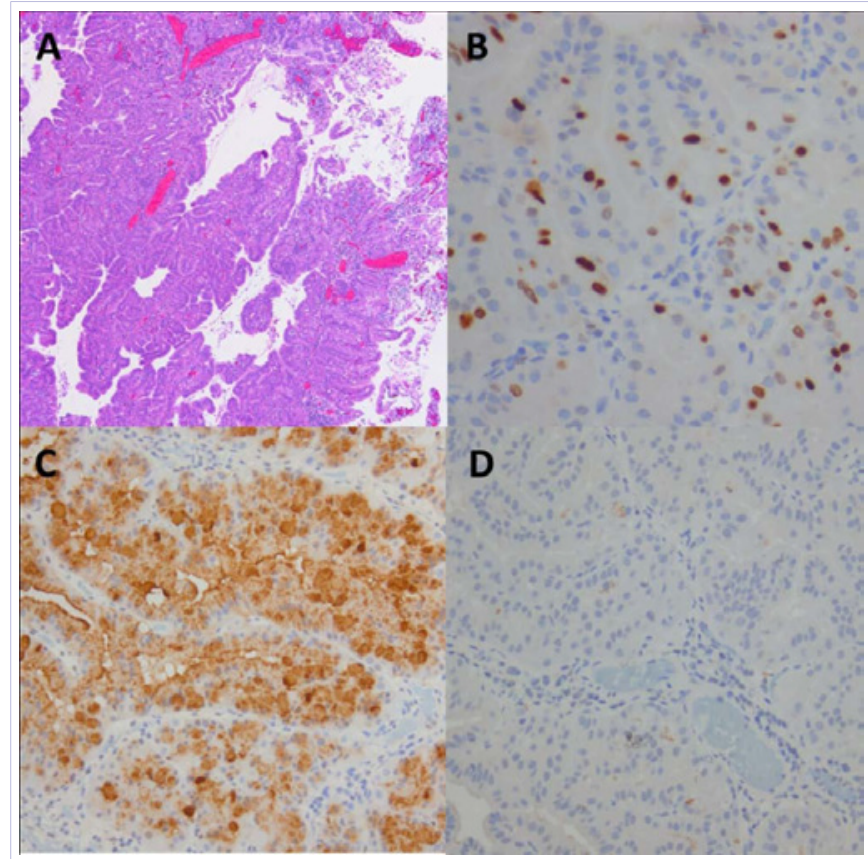

Figure 3: (a) HE section showed tubulovillous adenoma with middlehigh grade dysplasia, oxyphilic type. (b) Immunohistology showed KI67 $(30 \%+),(c)$ MUC5 (+), (d) CK20 (-).

\section{Discussion}

Duodenal subepithelial lesions are rare, with majority probably being neuroendocrine tumors [2]. Subepithelial lesions with umbilication are also rare, often regarded typical for ectopic pancreas [3]. Meanwhile, premalignant/ malignant transformation of ectopic gland might even be rarer [4]. Our case combined all three scenarios. The pop eye mucosal bulge with an umbilicus seemed to be a subepithelial lesion, whose diagnosis was most likely an ectopic pancreas according to its appearance before resection. However, HE staining of the specimen showed tubulovillous adenoma with middle-high grade dysplasia and finial pathological diagnosis was intraepithelial neoplasia originating from ectopic gland.

The reported incidence of ectopic pancreas ranges from $0.25 \%$ to $1.2 \%$ [5], mostly found in duodenum (29.3\%), stomach (27.4\%), jejunum (15.7\%), ileum (5.9\%), Meckel's diverticulum (5.1\%), and gallbladder (2.7\%) [6]. Chances are that similar pathological changes such as acute pancreatitis or cyst formation may occur in ectopic pancreas, but dysplasia and malignant degeneration rarely develops [7]. In our case, the intraepithelial neoplasia was hidden under normal epithelium of duodenum; with a "pop eye" left on it's the surface. Without the resection, it might never be suspected and have a chance of malignancy, causing much more serious problems to the patient.
Accurate diagnosis of subepithelial tumors before resection remains an arduous problem. Endoscopic ultrasound (EUS) does not contribute to the differential diagnosis as well in subepithelial tumors as in epithelial lesions. Tissue biopsy and sampling before operation are also ineffective due to their high false negative rate [8]. Thus, best treatment for duodenal subepithelial lesions is unclear due to the limited experience. However, our case highlights the importance of endoscopic intervention, rather than pure observation and surveillance, given the malignant potential of a seemingly benign lesion.

\section{Authors' Contributions}

TY Chen and XC Zhang contributed the same in the writing of the manuscript. MY Cai was involved with formation of the study concept and design, drafting of the manuscript and the literature review. XW Ge contributed to the pathological and immunohistochemistry examinations. PH Zhou carried out the endoscopic operation on the patient and was the main contributor in the writing of the manuscript. All authors have read and approved the final version of the manuscript.

\section{References}

1. Pittayanon R, Imraporn B, Rerknimitr R, Kullavanijaya P. Advances in diagnostic endoscopy for duodenal, including ampullary, adenoma. Dig Endosc. 2014;26: 10-5. DOI: 10.1111/den.12244.

2. Kojima T, Takahashi H, Parra-Blanco A, Kohsen K, Fujita R. Diagnosis of submucosal tumor of the upper GI tract by endoscopic resection. Gastrointest Endosc. 1999; 50(4):516-522. DOI: doi.org/10.1016/ S0016-5107(99)70075-1.

3. Lucena JF, Alvarez OA, Gross GW. Endoscopic resection of heterotopic pancreas of the minor duodenal papilla: case report and review of the literature. Gastr http://www.giejournal.org/article/S00165107(07)03059-3/abstractointest Endosc. 1997; 46(1): 69-72.

4. Eum JS, Kim GH, Park CH, Kang DH, Song GA. A remnant cystic duct cancer presenting as a duodenal submucosal tumor. Gastrointest Endosc.2008;67(6):975-976. DOI: doi.org/10.1016/j.gie.2007.11.010.

5. Fukino N, Oida T, Mimatsu K, Kuboi Y, Kida K. Adenocarcinoma arising from heterotopic pancreas at the third portion of the duodenum. World J Gastroenterol. 2015;21(13):4082-4088. doi: 10.3748/wjg. v21.i13.4082.

6. Inoue Y, Hayashi M, Arisaka Y, Higuchi K, Egashira Y, Tanigawa N. Adenocarcinoma arising in a heterotopic pancreas (Heinrich type III): a case report. J Med Case Rep. 2010;6(4):39. DOI: 10.1186/17521947-4-39.

7. Ginori A, Vassallo L, Butorano MA, Bettarini F, Di Mare G, Marrelli D. Pancreatic adenocarcinoma in duodenal ectopic pancreas: a case report and review of the literature. Pathologica. 2013;105(2):56-58.

8. Sumiyoshi T, Shima Y, Okabayashi T, Kohsaki T, Kigi A, Iwata J, et al. Heterotopic Pancreas in the Common Bile Duct, with a Review of the Literature. Intern Med. 2014;53(23):2679-2682. 